\title{
Foreword: Special section on performance evaluation methodologies
}

\author{
Jean Mairesse
}

Received: 21 December 2010 / Accepted: 21 December 2010 / Published online: 6 January 2011

(C) Springer Science+Business Media, LLC 2011

This special section contains a selection of three papers whose original versions were presented at the Valuetools conference, held in Pisa, Italy, from October 20 to 22, 2009. All three papers have gone through a new and complete reviewing process. They were thoroughly reworked, enlarged, and improved with respect to the conference versions.

The three papers of the section provide an overview of various mathematical models of stochastic discrete event systems. The paper by M. Draief and A. Ganesh studies the spread of an infection on a graph, and the effect of the graph topology on the speed of contamination. The paper by R. Jain, S. Juneja, and N. Shimkin tackles a specific optimisation problem based on a queueing model in which customers can choose their arrival time. Finally, N. Gast and B. Gaujal propose a framework to study the asymptotic limit of large Markov decision processes. The second paper received the Best Paper Award of the Valuetools conference, and the third one received the Best Student Paper Award.

The aim of the VALUETOOLS conference is to gather researchers from various communities working on performance evaluation and tools. The conference in Pisa was the fourth of the VAluetools conferences. Precisely 70 papers were submitted, and 27 of them were selected for presentation at the conference. The 8 best papers were selected for possible publication in two journals: Discrete Event Dynamic Systems and Performance Evaluation.

I thankfully acknowledge the help of the sponsors of VALUETOoLs: ICST/Createnet, ACM, ACM Sigmetrics, the Society for Modelling and Simulation International, and MIMOS (Movimento Italiano Modellazione e Simulazione). I am grateful to all the reviewers for their time, effort and useful suggestions. And last but not least, my special thanks go to the members of the Steering and Organizing Committees, and in particular to Giovanni Stea, the very efficient Conference General Chair.

J. Mairesse $(\bowtie)$

LIAFA, CNRS and University Paris 7, Paris, France

e-mail: mairesse@liafa.jussieu.fr 